\title{
Erratum to: Close intramolecular sulfur-oxygen contacts: modified force field parameters for improved conformation generation
}

Dmitry Lupyan · Yuriy A. Abramov •

Woody Sherman

Published online: 19 March 2013

(C) Springer Science+Business Media Dordrecht 2013

Erratum to: J Comput Aided Mol Des (2012)

\section{6:1195-1205}

DOI 10.1007/s10822-012-9610-x

The original article is correct as printed. However, the supporting supplementary information was not deposited with the original article and is provided here.

The online version of the original article can be found under doi:10.1007/s10822-012-9610-x.

Electronic supplementary material The online version of this article (doi:10.1007/s10822-013-9633-y) contains supplementary material, which is available to authorized users.

D. Lupyan · W. Sherman $(\bowtie)$

Schrodinger Inc., 120 West Forty-Fifth Street, 17th Floor,

New York, NY 10036, USA

e-mail: Woody.Sherman@schrodinger.com

Y. A. Abramov ( $\bowtie)$

Pfizer Global Research and Development, Eastern Point

Road, Groton, CT 06340, USA

e-mail: Yuriy.A.Abramov@pfizer.com 